\title{
Philosophiques
}

\section{Alain Badiou, Le Nombre et les nombres, Paris, Éditions du Seuill (coll. « Des Travaux »), 1990, 278 pages.}

\section{Yvon Gauthier}

Volume 20, numéro 1, printemps 1993

URI : https://id.erudit.org/iderudit/027213ar

DOI : https://doi.org/10.7202/027213ar

Aller au sommaire du numéro

Éditeur(s)

Société de philosophie du Québec

ISSN

0316-2923 (imprimé)

1492-1391 (numérique)

Découvrir la revue

Citer ce compte rendu

Gauthier, Y. (1993). Compte rendu de [Alain Badiou, Le Nombre et les nombres, Paris, Éditions du Seuil (coll. « Des Travaux »), 1990, 278 pages.] Philosophiques, 20(1), 204-205. https://doi.org/10.7202/027213ar d'utilisation que vous pouvez consulter en ligne.

https://apropos.erudit.org/fr/usagers/politique-dutilisation/ 
Alain Badiou, Le Nombre et les nombres, Paris, Éditions du Seuil (coll. "Des Travaux"), 1990, 278 pages.

\section{par Yvon Gauthier}

Dans ce dernier ouvrage, l'auteur poursuit la méditation qu'il avait entreprise avec l'Être et lévénement (Paris, Éditions du Seuil, 1988) dont nous avons déjà rendu compte (Dialogue XXIX, n 3, 1990, 471-473). Si, dans son travail précédent. l'Auteur avait pris prétexte de la théorie des ensembles pour dérouler une longue suite de méditations et de réflexions sur l'ontologie, c'est maintenant la théorie des nombres surréels qui est, dans les mots de l'Auteur. «l'extension tout à fait naturelle au concept de nombre de la vocation ontologique de la théorie des ensembles » (p. 275). Les nombres surréels ont été inventés par Conway (voir On Numbers and Games, London, Academic Press, 1976) en s'inspirant de la théorie des coupures de Dedekind, théorie qui a servi à la constitution de la théorie des nombres reels - on trouvera une introduction succincte à la théorie des nombres surréels dans nos Méthodes et concepts de la logique formelle (Montréal, P.U.M., 1978, p. 207-212).

Dans une première partie, A. Badiou retrace les « Généalogies » que sont à ses yeux Frege, Dedekind, Peano et Cantor pour ensuite aborder les «Concepts » que sont les multiplicités naturelles avant d'accéder à l'ontologie du Nombre, dont la majuscule signifie la dignité d'un concept ontologique ou poétique premier (p. I34). C'est ici que l'on trouvera l'essentiel des réflexions de l'Auteur, son concept de « sous-nombre » qui fait pendant au « sur-nombre » qu'est le nombre surréel. Le théorème fondamental (p. I74 et s.) de l'ontologie du nombre n'est que la reprise du théoreme d'existence fondamental que l'on trouve dans H. Gonshor, An Introduction to the Theory of Surreal Numbers, (London, Cambridge University Press, I986, p. 4).

Or l'Auteur se donne beaucoup de mal pour définir les notions de section et d'ordinal, alors que Gonshor, comme tout le monde, utilise simplement la notion de segment initial de la suite des nombres naturels (ou des ordinaux pour l'induction transfinie).

Ce qui fascine l'Auteur, c'est l'ontologie secrète du nombre, signe ou nom du multiple. Son ontologie mathématique fait fi des mathématiques sans ontologie et ne s'embarrasse guère des distinctions formelles, aussi bien dans la partie historique (l'arithmétique de Peano est présentée comme une théorie du second ordre où elle est catégoriquel que dans l'évaluation de la situation contemporaine - l'Auteur fait beaucoup ètat du livre de Gonshor, mais il ignore l'ouvrage de N.L. Alling, Foundations of A nalysis over Surreal Number Ficlds, (Amsterdam, North-Holland Mathematics Studies 141, 1987), qui comporte des théorèmes importants, par exemple sur l'hyperconvergence des séries de puissances formelles.

Une quatrième partie sur « Les dimensions opératoires » et une «Conclusion » viennent compléter cet itinéraire méditatif sur l'essence du Nombre, qui est une forme de l'Être (p. 26I). Cette ontologie platonisante ne se donne pas les moyens de sa réalisation. L'effort matériel est ici plus considérable que l'effort formel et le commentaire littéral et littèraire d'une thèorie 
mathématique sans visée fondationnelle et en faisant l'économie de la logique mathématique n'intéressera pas le philosophe des mathématiques; tout au plus intriguera-t-il le philosophe tout court qui ne dédaigne pas le chant multiple des voix entremêlées de la philosophie et de la poésie, de Platon à Hejdegger ou encore d'Aristote à Mallarmé pour qui le nombre est à la fois nombrant et nombré.

Département de philosophie

Université de Montréal 